\title{
Neural Transdifferentiation: MAPTau Gene Expression in Breast Cancer Cells
}

\author{
E Lara-Padilla ${ }^{1}$, A Miliar-Garcia ${ }^{2}$, M Gomez-Lopez ${ }^{2}$, P Romero-Morelos ${ }^{3}$, CI \\ Bazan-Mendez ${ }^{4}$, A Alfaro-Rodriguez ${ }^{5}$, M Anaya-Ruiz ${ }^{6}, \mathrm{~K}_{\text {Callender }}{ }^{7}, \mathrm{~A} \mathrm{Carlos}^{8}$, \\ C Bandala ${ }^{5 *}$
}

\begin{abstract}
Background: In tumor cells, aberrant differentiation programs have been described. Several neuronal proteins have been found associated with morphological neuronal-glial changes in breast cancer (BCa). These neuronal proteins have been related to mechanisms that are involved in carcinogenesis; however, this regulation is not well understood. Microtubule-associated protein-tau (MAP-Tau) has been describing in BCa but not its variants. This finding could partly explain the neuronal-glial morphology of BCa cells. Our aim was to determine mRNA expression of MAP-tau variants 2,4 and 6 in breast cancer cell lines. Materials and Methods: Cultured cell lines MCF-10A, MDA-MB-231, SKBR3 and T47D were observed under phase-contrast microscopy for neural morphology and analyzed for gene expression of MAP-Tau transcript variants 2, 4 and 6 by real-time PCR. Results: Regarding morphology like neural/glial cells, T47D line shown more cells with these features than MDA-MB-231 and SKBR. In another hand, we found much greater mRNA expression of MAP-Tau transcript variants 2 , and to a lesser extent 4 and 6 , in T47D cells than the other lines. In conclusion, regulation of MAPTau could bring about changes in cytoskeleton, cell morphology and motility; these findings cast further light on neuronal transdifferentiation in BCa.
\end{abstract}

Keywords: Breast cancer - neural transdifferentiation - neuron/glial-like cell - microtubule-associated protein tau

Asian Pac J Cancer Prev, 17 (4), 1967-1971

\section{Introduction}

Breast cancer $(\mathrm{BCa})$ is the most prevalent worldwide oncologic disease in women. BCa incidence and mortality has increased in Mexico in the last years. (Perez-Santos and Anaya-Ruiz, 2013; Anaya-Ruiz et al., 2014; LeonHernandez et al., 2014; Soto-Perez-de-Celis and ChavarriGuerra, 2016). Cell transdifferentiation is a phenomenon of genetic reprogramming and protein expression change; however, transdifferentiated cells could show morphological and phonotypical changes of neuronal linage despite, their linage is different (Cho et al., 2005; Bauer et al., 2012; Zhang et al., 2010; Palafox et al., 2012).

It has been shown that $\mathrm{BCa}$ cell lines have been found specific markers of neuronal / glial type lineage, indicating that aberrant transdifferentiation is a phenomenon that occurs in this type of cancer (Zhang et al., 2010). Regarding finding to neuronal / glial morphology phenotype, described in the line MDA-MB-435 (lung cell lines derived from lung metastases), has been shown the presence of polygonal soma (cell body), extended dendrites and axons, and cell-cell communication at synapses (Zhang et al., 2010). Different BCa cell lines show expression of a wide number of neuronal proteins such as: Synaptic Vesicle Protein 2 (SV2A) (Bandala et al., 2012), beta-tubulin class III gene (TuBB3), nestin, (Zhang et al., 2010; Kanojia et al., 2015), SNAP23, vesicle-associated membrane protein 7 (VAMP7), Syntaxin4, 8 (Williams et al., 2014; Bassett et al., 2008), microtubule-associated protein tau (MAP-Tau) (Honig, et al., 2014), synuclein gamma (Ahmad et al., 2007; Guo et al., 2007), synaptotagmin (Sreenath et al., 2005), synapsin 1 (Sadeqzadeh et al., 2011).

Further, cytoskeleton and cytoskeleton-associated proteins are important for cell reprogramming processes (Cheng et al., 2013). There are many cytoskeleton associated proteins, one of them proteins are the Microtubule-associated protein (MAP-Tau) which is a family of proteins that regulate the microtubule dynamics (Zhou et al., 2015). Interestingly, MAP-Tau is widely 
distributed in central nervous system, and recent data suggest that might also play a physiological role in dendrites, but its most abundant in axons. MAP-Tau is a multifunctional protein and its function depends on the protein phosphorylation state. The phosphorylation of MAP-Tau could result in its ability to bind to microtubules, and alteration in these modifications results in neurodegenerative diseases (Goedert et al., 1988; Goedert et al., 1989; Shin et al., 1991; Mietelska-Porowska et al., 2014). In the other hand, MAP-Tau proteins are accepted as marker in paclitaxel response in breast cancer showing that MAP-Tau could repress the activity of taxane drugs (Zhou et al., 2015). MAP-Tau and others molecules have been detected in different cancer types and could be used as molecular markers of cancer and neural transdifferentiation and, like MAP-Tau, could explain transformation, immune-evasion, carcinogenesis and metastasis (Goedert et al., 1988; Goedert et al., 1989; Shin et al 1991; Chapin \& Bulinski, 1992; Harada et al., 1994; Paris et al ., 2011; Zang et al., 2011; Ksiazkiewicz et al., 2012; Palafox et al., 2012; Kotoula et al., 2013).

Few studies related microtubule-associated proteins expressions with BCa (Rody et al., 2007; Bauer et al., 2012; Ikeda et al., 2012; Moleans et al., 2011; Penteroudakis et al., 2011; Sadi et al., 2011; Kolacinska et al., 2012; Leontovich et al, 2012; Endo et al., 2013; Kotoula et al., 2013), that proceed most commonly from the inner lining of milk ducts or the lobules that supply the ducts with milk (Sariego 2010). But, in this research we studied 3 different variants of MAP-Tau in different $\mathrm{BCa}$ cell lines.

\section{Materials and Methods}

\section{Cell culture}

T47D, SKBR3 and MDA-MB-231 cell lines were obtained from ATCC (Manassa, VA). MCF-10A cells were gifted from Dr. Juan Pedro Luna Arias, CINVESTAV.T47D were cultured in RPMI-1640 (GIBCO). MCF-10A were cultured in DMEM F12 (GIBCO) supplemented with 4.18 $\mu \mathrm{f} / \mathrm{ml}$ insulin, $10 \mu \mathrm{g} / \mathrm{ml}$ HEGF, $0.4 \mu \mathrm{g} / \mathrm{ml}$ Hydrocortisone. MDA-MB-231 was cultured in DMEM high glucose (Hyclon, Logan, Utah). All cell lines were supplemented with $10 \%$ FBS (GIBCO) and $1 \%$ penicillin/streptomycin (SIGMA). Cells were grown as monolayers under standard conditions at $37^{\circ} \mathrm{C}$ in humidified atmosphere containing 5 $\% \mathrm{CO}_{2}$ and $95 \%$ air. The cells were cultured in BD Falcon $75 \mathrm{~cm}^{2}$ Cell Culture Flask, $250 \mathrm{ml}$ for morphologic and genic expression analysis.

\section{Cell culture morphologic analysis}

The moment that cell cultures reach confluence was regarded time zero and they stayed in incubation until 6 days. Then cell were observed daily to start to $24 \mathrm{~h}$, and in 6 day they were photographed. The morphology of cells on slides was observed with an inverted Olympus MIC-D Digital Microscope with phase contrast, imaging acquisition system and 40x objective lens.

\section{RNA extraction and RT-PCR assays}

Total RNA from the tissue adipose was isolated using TRIzol Reagent according to the protocol of manufacturer (Life Technologies, USA). Isolated RNA was quantified using the GENESYS 10 Series spectrophotometer (Thermo Scientific, USA) and $5 \mu \mathrm{g}$ of RNA were separated on a $1.0 \%$ agarose gel containing ethidium bromide in MOPS buffer. Running buffer and gel contained $0.2 \mathrm{M}$ formaldehyde. To avoid trace amounts of DNA contamination, RNA samples were treated with amplification grade DNase I (Invitrogen) before reverse transcription. All RNA samples were stored at $-70{ }^{\circ} \mathrm{C}$ in RNA elution solution until further use. We used $0.5 \mu \mathrm{g}$ of RNA for reverse-transcription with random hexamers in 20- $\mu$ l reaction volume using Transcriptor First Strand cDNA Synthesis kit (Roche Diagnostics). The reactions were performed in Eppendorf Mastercycler thermocycler (Eppendorf, Mexico). The amplified cDNA was quantified on a photometer at $260 \mathrm{~nm}$. RT-PCR reactions were carried out using the Human Universal ProbeLibrary (Roche Diagnostics). Specific oligonucleotide primers for MAPTau transcript variants 2, 4 and 6 were originally generated by using the online assay design software (ProbeFinder: http://www.universal-probelibrary.com) and the primer sequence for each gene that is shown in Table 1 . The 20 $\mu$ l-reaction mixture contained 1x LightCycler TaqMan Master reaction mixture (Roche Diagnostics), $200 \mathrm{nM}$ of each primer, $100 \mathrm{nM}$ of Universal ProbeLibrary probe, $0.5 \mathrm{U}$ LightCycler Uracil-DNAGlycosylase and $2 \mu \mathrm{l}$ of standard DNA in appropriate dilution.

The amplification was performed in borosilicate glass capillaries (Roche Diagnostics). The RT-PCR assay included a standard curve of four serial dilution points for each gene and samples were normalized with the endogenous control 18S. Data were analyzed by LightCycler software.

\section{Statistics}

The percentage of cells with different morphology was determined for observed field and was plotted. For compare mRNA expression was utilized Kruskal-Wallis One Way Analysis of Variance on Ranks. Statistical Analysis was performed with SPSS v17 for Windows XP (SPSS, UK, Ltd, Working, UK) and SigmaPlot for Windows v11.0. $\mathrm{P}<0.05$ was regarded as significant.

Table 1. Primer Sets for MAPT used in Real-time PCR with Designs Based on Ensemble Transcript ID of the Human Probe Library

\begin{tabular}{cccc}
\hline Gene & $\begin{array}{c}\text { Nucleotide sequence acces- } \\
\text { sion number }\end{array}$ & Left primer 5'-3' & Right primer 5'-3' \\
\hline Transcript variant 2 & NM_005910.5 & gatcccagaaggaaccacag & ttttactgaccatgcgagctt3 \\
Transcript variant 4 & NM_001134364.1 & accacagccaccttctcct & cagccatcctggttcaaagt \\
Transcript variant 6 & NM_001123066.3 & tctcttctgtcacttcccgaac & gcgatcttcgttttaccatca \\
\hline
\end{tabular}




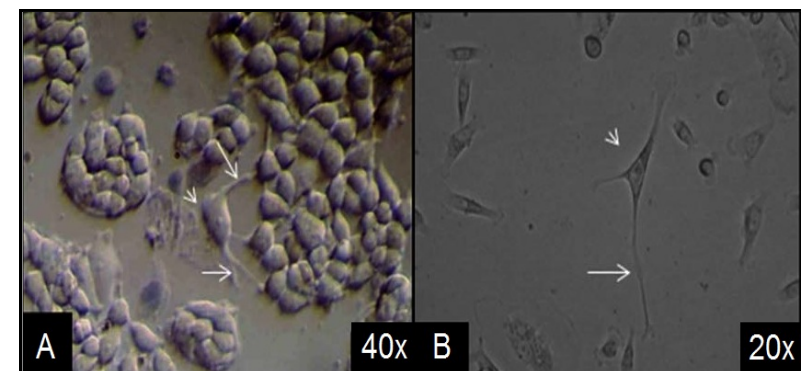

Figure 1. BCa-Cell Lines Differentiate Into Neuron/ Glial-Like Cell. Phases-contrast microscopy of cell culture. A) T47D, B) MDA-MB-231. Arrows indicate neuraltransdifferentiation cell
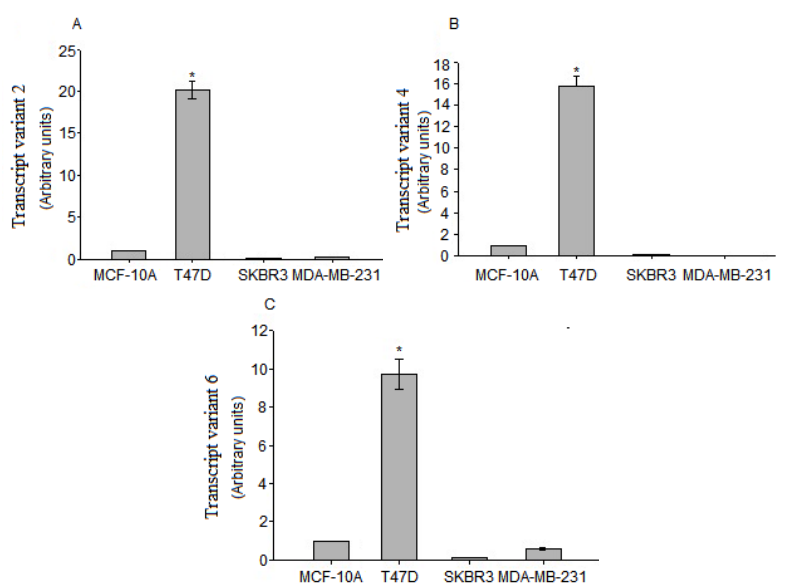

Figure 2. The Relative Expression Level of genes Transcript Variants 2, 4 and $6(\mathrm{~A}, \mathrm{~B}, \mathrm{C})$ of MAP-Tau was Determined After Normalization Against the 18S Internal Control for Each Sample. Data represent the mean \pm SD, *P vs. control (MCF-10A)

\section{Results}

Presence of cell transdifferentiation in breast cancer

With the aim to know if there is cell transdifferentiation in $\mathrm{BCa}$ and that there not is eventful simple dedifferentiation or cell function loss, the cell morphology was explored. It was observed among cancer cells of habitual morphology ones with different aspect, like a neural cells, which were common in TD47D (Figure. 1A) and MDA-MB-231 (Figure. 1B). In each case, the cells showed a uniform morphology until third day of culture, and then cells changed their aspect with extensions like axons of neural cells, feature more appreciable when the cells were dispersed, suggesting a glial/neural transdifferentiation in phenotype of BCa lines. Neuron-like cells formed a subpopulation that reach $4 \%$ of observed total fields in T47D cell line. The transdifferentiation was greater in T47D that MDA-MB-231 (37.8\%) and MDA-MB-453 $(60.1 \%)$ lines.

\section{Neural gene expression in breast cancer and non-tumor} cell lines

We analyzed the MAP-Tau mRNA transcript variants in three cancer cell lines and compared with epithelial cells, non-transformed derived from human fibrocystic mammary tissue, MCF-10A (Figure 2). The expression of different transcript variants was founded in each cell line. There was not expression difference between SKRB3,

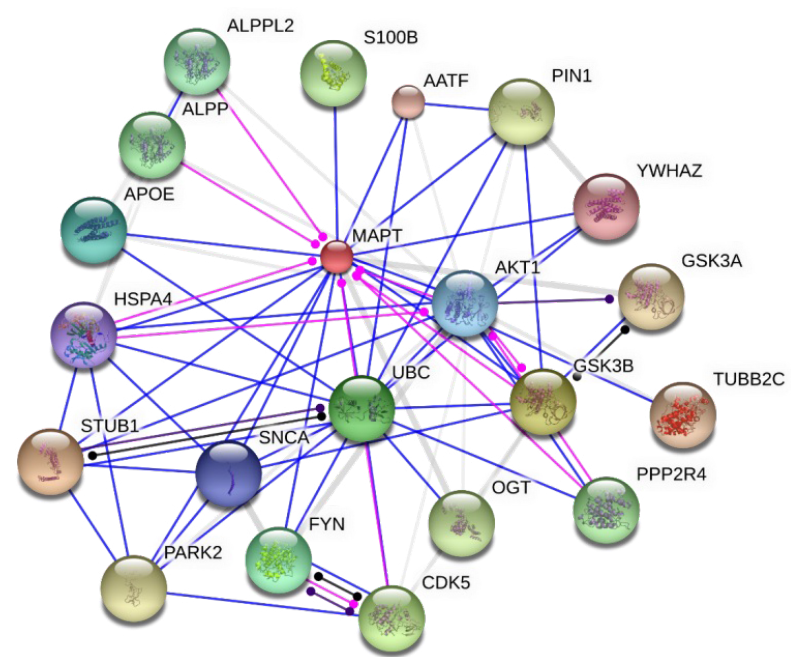

Figure 3. STRING Interaction Network Showing Association between Differentially Expressed Proteins. The interaction map was generated using default settings (Medium confidence of 0.4 and 7 criteria for linkage: activation, Inhibition, binding, phenotype, catalysis, post-transl. $\mathrm{m}$, reaction, expression). Interactions are represented as follows: binding in blue, Post-translational modification in pink, expression in green, catalysis in violet and reaction in black

MDA-MB-231 and non-tumor cell line for MAP-Tau transcript variants 2, 4 and 6 , however, the expression was higher in T47D respect to the others cells $(\mathrm{P}<0.05)$. Furthermore, variant 2 shown more mRNA expression the variant 4 and 6 .

\section{Protein Network Analysis}

STRING network analysis of protein-protein interactions was performed to identify functionally linked proteins and determine the potential biological processes affected [PMID:12519996]. The network is presented under confidence view, whereby stronger associations are represented by thicker lines or edges and vice versa, whereas proteins are represented as nodes. All gene symbols were derived from the HUGO Gene Nomenclature Committee (HGNC) (http://www. genenames.org). Figure 3 shows the interaction between 20 identified proteins and the additional interactions. Fourteen proteins were found to be linked for binding with MAP-Tau and six proteins for Post-translational modification. The interaction patterns will be helpful to have a better understanding of the protein functional activities. As the identified proteins are involved in the modulation of neuronal apoptosis process and cell growth (AATF and FYN), but also but also proteins associated with phosphorylation, ubiquitination and increase levels of tau protein (S100B, PIN1, STUB1, CDK5, AKT1, TUBB2C, and GSK3A).

\section{Discussion}

Cancer tumors usually are apparently dedifferentiated cell growths, which form a mosaic of disorganized, undifferentiated cell subpopulations that express noncharacteristic proteins of original tissue. Interestingly, the $\mathrm{BCa}$ cell underwent a differentiation process into neuron/glial-like cell, apparently changing its phenotypic 
aspect, and perhaps this could be regarding with epigenetic processes (McPherson et al., 2000; Battula et al., 2012; Palafox et al 2012). Neuronal / glial phenotype morphology was described in the MDA-MB-435 line (lung cell lines derived from lung metastases): such as the presence of polygonal soma (cell body), extended dendrites and axons, and cell-cell communication at synapses (Zhang et al., 2010), are similar to ours results in MDA-MB-231 line, MDA-MB-453, T47D.

We found MAP-Tau expression 2,4 and 6 transcript variants, which ones, interestingly are essential in neurogenesis process, stabilizing microtubules growth (Shin et al., 1991; Goedert et al., 1988; Goedert et al., 1989; Lim \& Halpain, 2000; Ray et al., 2011; BarnedaZahonero et al., 2012). These proteins suggest a neural nature of Neural-like cell founded in this study, because products of paralogous genes in rat and mouse were be associated with neuron-specific cytoskeletal proteins that are enriched in dendrites, implicating a role in determining and stabilizing dendritic shape during neuron development (Barneda-Zahonero et al., 2012). The regulation of MAP-Tau could bring changes in cytoskeleton and cell morphology and motility, which result unless in transdifferentiation look (Bengtsson et al., 1993; Goedert et al., 1988; Goedert et al., 1989; Yang et al., 2008; Barrachina and Ferrer, 2009). Our results showed that MAP-Tau transcript variants are related with $\mathrm{BCa}$, but may be associated with positive estrogen alpha receptors (Endo et al., 2013), in the other hand, recently has been found that MAP-Tau could be associated to kinesin-1 which is a molecular motor that transport different molecules across the microtubules, and interestingly, this Kinesine- 1 binds to the same microtubule region were paclitaxel binds and act, and may be, because of that MAP-Tau proteins are associated with poor prognosis and paclitaxel tumor resistance (Seeger and Rice, 2010), but other results are controversial (Honing et al., 2014). Cell transdifferentiation could have an important role, joint to RANK (Palafox et al 2012), ganglioside GD2 (Battula et al., 2012) or epigenetic causes (BarnedaZahonero et al., 2012) and such as in case of human adult peripheral blood mononuclear cells could be induced to transdifferentiate into neural precursor cells and retinal progenitor cells in vitro (Liu et al., 2011). In conclusion, regarding with the expression of MAP-Tau transcripts and with its function in neurogenesis, we suggest an apparently transdifferentiation, or a reprogramming process in $\mathrm{BCa}$ cell lines; MAP-Tau variant 2 has been more expressed than variant 4 and 6 in $\mathrm{BCa}$ cell lines.

\section{References}

Ahmad M, Attoub S, Singh MN, Martin FL, El-Agnaf OM (2007). Gamma-synuclein and the progression of cancer. FASEB J, 21, 3419-30.

Anaya-Ruiz M, Vallejo-Ruiz V, Flores-Mendoza L, Perez-Santos M (2014). Female breast cancer incidence and mortality in Mexico, 2000-2010. Asian Pac J Cancer Prev, 15, 1477-9.

Bandala C, Miliar-García A, Mejía-Barradas CM, et al (2012) Synaptic vesicle protein 2 (SV2) isoforms. Asian Pac J Cancer Prev, 13, 5063-7.

Barneda-Zahonero B, Roman-Gonzalez L, Collazo O, Mahmoudi
T, Parra M (2012). Epigenetic regulation of B lymphocyte differentiation, transdifferentiation, and reprogramming. Comp Funct Genomics, 2012, 564381.

Barrachina M, Ferrer I (2009). DNA methylation of Alzheimer disease and tauopathy-related genes in postmortem brain. $J$ J Neuropathol Exp Neurol, 68, 880-1.

Bassett T, Harpur B, Poon HY, Kuo KH, Lee CH (2008). Effective stimulation of growth in MCF-7 human breast cancer cells by inhibition of syntaxin 18 by external guide sequence and ribonuclease P. Cancer Lett, 272, 167-75.

Battula VL, Shi Y, Evans KW, et al (2012). Ganglioside GD2 identifies breast cancer stem cells and promotes tumorigenesis. J Clin Invest, 122, 2066-8.

Bauer JA, Chakravarthy AB, Rosenbluth JM, et al (2012). Identification of markers of taxane sensitivity using proteomic and genomic analyses of breast tumors from patients receiving neoadjuvant paclitaxel and radiation. Clin Cancer Res, 16, 681-0.

Bengtsson T, Jaconi ME, Gustafson M, et al (1993). Actin dynamics in human neutrophils during adhesion and phagocytosis is controlled by changes in intracellular free calcium. Eur J Cell Biol, 62, 49-8.

Chapin SJ, Bulinski JC (1992). Microtubule stabilization by assembly-promoting microtubule-associated proteins: a repeat performance. Cell Motil Cytoskeleton, 23, 236-3.

Cheng Y, Gaughan J, Midic U et al (2013). Systems genetics implicates cytoskeletal genes in oocyte control of cloned embryo quality. Genetics, 193, 877-6.

Cho KJ, Trzaska KA, Greco SJ, et al (2005). Neurons derived from human mesenchymal stem cells show synaptic transmission and can be induced to produce the neurotransmitter substance P by interleukin-1 alpha. Stem Cells, 23, 383-1.

Endo Y, Toyama T, Takahashi S et al (2013). miR-1290 and its potential targets are associated with characteristics of estrogen receptor alpha-positive breast cancer. Endocr Relat Cancer, 20, 91-2.

Goedert M, Wischik CM, Crowther RA, Walker JE, Klug A (1988). Cloning and sequencing of the cDNA encoding a core protein of the paired helical filament of Alzheimer disease: identification as the microtubule-associated protein tau. Proc Natl Acad Sci USA, 85, 4051-5.

Goedert M, Spillantini MG, Jakes R, Rutherford D, Crowther RA (1989). Multiple isoforms of human microtubule-associated protein tau: sequences and localization in neurofibrillary tangles of Alzheimer's disease. Neuron, 3, 519-26.

Guo J, Shou C, Meng L, et al (2007). Neuronal protein synuclein gamma predicts poor clinical outcome in breast cancer. Int J Cancer, 121, 1296-305.

Harada A, Oguchi K, Okabe S (1994). Altered microtubule organization in small-calibre axons of mice lacking tau protein. Nature, 369, 488-1.

Honig A, Gehrmann M, Kranke P, et al. (2014). Microtubuleassociated protein tau correlates with estrogen receptor status but not with in vitro paclitaxel sensitivity in primary breast cancer. Eur J Gynaecol Oncol, 35, 503-7.

Ikeda H, Taira N, Hara F et al. (2010). The estrogen receptor influences microtubule-associated protein tau (MAPT) expression and the selective estrogen receptor inhibitor fulvestrant downregulates MAPT and increases the sensitivity to taxane in breast cancer cells. Breast Cancer Res, 12, 43.

Kanojia D, Morshed RA, Zhang L, et al (2015). BIII-tubulin regulates breast cancer metastases to the brain. Mol Cancer Ther, 14, 1152-61.

Kolacinska A, Fendler W, Szemraj J et al (2012). Gene expression and pathologic response to neoadjuvant chemotherapy in 
breast cancer. Mol Biol Rep, 39, 7435-1.

Kotoula V, Kalogeras KT, Kouvatseas G et al (2013). Sample parameters affecting the clinical relevance of RNA biomarkers in translational breast cancer research. Virchows Arch, 462, 141-4.

Książkiewicz M, Markiewicz A, Zaczek AJ (2012). Epithelialmesenchymal transition: a hallmark in metastasis formation linking circulating tumor cells and cancer stem cells. Pathobiol, 79, 195-8.

Leon-Hernandez SR, Padilla EL, Algara AC, et al (2014). Relation of alcohol/tobacco use with metastasis, hormonal (estrogen and progesterone) receptor status and c-erbB2 protein in mammary ductal carcinoma. Asian Pac J Cancer Prev, 15, 5709-14.

Leontovich AA, Zhang S, Quatraro C et al (2012). Raf-1 oncogenic signaling is linked to activation of mesenchymal to epithelial transition pathway in metastatic breast cancer cells. Int J Oncol, 40, 1858-4.

Lim RW, Halpain S (2000). Regulated association of microtubule-associated protein 2 (MAP2) with Src and Grb2: evidence for MAP2 as a scaffolding protein. $J$ Biol Chem, 275, 20578-7.

Liu Q, Guan L, Huang B, et al (2011). Adult peripheral blood mononuclear cells transdifferentiate in vitro and integrate into the retina in vivo. Cell Biol Int, 35, 631-8.

McPherson K, Steel CM, Dixon JM (2000). ABC of breast diseases. Breast cancer-epidemiology, risk factors, and genetics. BMJ, 321, 624-8.

Mietelska-Porowska A, Wasik U, Goras M, Filipek A, Niewiadomska $G$ (2014). Tau protein modifications and interactions: their role in function and dysfunction. Int $J$ Mol Sci, 15, 4671-3.

Moelans CB, de Wegers RA, Monsuurs HN, Maess AH, van Diest PJ (2011). Molecular differences between ductal carcinoma in situ and adjacent invasive breast carcinoma: a multiplex ligation-dependent probe amplification study. Cell Oncol, 34, 475-2.

Palafox M, Ferrer I, Pellegrini P et al (2012). RANK induces epithelial-mesenchymal transition and stemness in human mammary epithelial cells and promotes tumorigenesis and metastasis. Cancer Res, 72, 2879-8.

Paris AJ, Snapir Z, Christopherson CD et al (2011). A polymorphism that delays fibrosis in hepatitis $\mathrm{C}$ promotes alternative splicing of AZIN1, reducing fibrogenesis. Hepatol, 54, 2198-7.

Pentheroudakis G, Batistatou A, Kalogeras KT et al (2011). Prognostic utility of beta-tubulin isotype III and correlations with other molecular and clinicopathological variables in patients with early breast cancer: a translational Hellenic Cooperative Oncology Group (HeCOG) study. Breast Cancer Res Treat, 127, 179-3.

Perez-Santos JL, Anaya-Ruiz M (2013). Mexican breast cancer research output, 2003-2012. Asian Pac J Cancer Prev, 14, 5921-3.

Ray B, Chauhan NB, Lahiri DK (2011). The "aged garlic extract:" (AGE) and one of its active ingredients S-allylL-cysteine (SAC) as potential preventive and therapeutic agents for Alzheimer's disease (AD). Curr Med Chem, 18, 3306-3.

Rody A, Karn T, Gätje R et al (2007). Gene expression profiling of breast cancer patients treated with docetaxel, doxorubicin, and cyclophosphamide within the GEPARTRIO trial: HER2, but not topoisomerase II alpha and microtubule-associated protein tau, is highly predictive of tumor response. Breast, 16, 86-3.

Sadeqzadeh E, Rahbarizadeh F, Ahmadvand D, et al (2011). Combined MUC1-specific nanobody-tagged PEG- polyethylenimine polyplex targeting and transcriptional targeting of tBid transgene for directed killing of MUC1 over-expressing tumour cells. J Control Release, 156, 85-91.

Sadi AM, Wang DY, Youngson BJ et al (2011). Clinical relevance of DNA microarray analyses using archival formalin-fixed paraffin-embedded breast cancer specimens. BMC Cancer, 11, 1-3.

Sariego J (2010). Breast cancer in the young patient. Am Surg, 76, 1397-0.

Seeger MA, Rice SE (2010). Microtubule-associated protein-like binding of the kinesin- 1 tail to microtubules. J Biol Chem, 285, 8155-62.

Shin RW, Iwaki T, Kitamoto T, Tateishi J (1991). Hydrated autoclave pretreatment enhances tau immunoreactivity in formalin-fixed normal and Alzheimer's disease brain tissues. Lab Invest, 64, 693-702.

Soto-Perez-de-Celis E, Chavarri-Guerra Y (2016). National and regional breast cancer incidence and mortality trends in Mexico 2001-2011: Analysis of a population-based database. Cancer Epidemiol, 41, 24-33.

Sreenath AS, Kumar KR, Reddy GV, et al (2005). Evidence for the association of synaptotagmin with glutathione S-transferases: implications for a novel function in human breast cancer. Clin Biochem, 38, 436-43.

Williams KC, McNeilly RE, Coppolino MG (2014). SNAP23, Syntaxin 4, and vesicle-associated membrane protein 7 (VAMP7) mediate trafficking of membrane type 1-matrix metalloproteinase (MT1-MMP) during invadopodium formation and tumor cell invasion. Mol Biol Cell, 25, 2061-70.

Zhang H, Kouadio A, Cartledge D, Godwin AK (2011). Role of gamma-synuclein in microtubule regulation. Exp Cell Res, 317, 1330-9.

Zhang Q, Fan H, Shen J, Hoffman RM, Xing HR (2010). Human breast cancer cell lines co-express neuronal, epithelial, and melanocytic differentiation markers in vitro and in vivo. PLoS One, 5, 9712.

Zhou J, Qian S, Li H et al (2015). Predictive value of microtubule-associated protein Tau in patients with recurrent and metastatic breast cancer treated with taxane-containing palliative chemotherapy. Tumour Biol, 36, 3941-7. 